\title{
Mortality associated with acute kidney injury in COVID-19 admissions to a high dependency/intensive care setting
}

\author{
Authors: Hina Iftikhar, ${ }^{\mathrm{A}}$ Seema Alaee, ${ }^{\mathrm{A}}$ Jessica Bennett, ${ }^{\mathrm{A}}$ Rachel Kaminski, ${ }^{\mathrm{A}}$ David Windsor ${ }^{\mathrm{A}}$ and Charles Sharp ${ }^{\mathrm{A}}$
}

\begin{tabular}{|c|c|c|c|c|c|}
\hline & $\begin{array}{l}\text { CPAP only, } \\
n=71\end{array}$ & $\begin{array}{l}\text { IMV only, } \\
n=34\end{array}$ & $\begin{array}{l}\text { CPAP and IMV, } \\
n=24\end{array}$ & $\begin{array}{l}\% \text { of cohort } \\
\text { with AKI }\end{array}$ & $\begin{array}{l}\% \text { of entire } \\
\text { ITU/HDU cohort }\end{array}$ \\
\hline Patients with AKI (\%) & $30(42 \%)$ & $4(12 \%)$ & $9(38 \%)$ & $43(100 \%)$ & $43(33 \%)$ \\
\hline \multicolumn{6}{|l|}{ Rockwood Clinical Frailty Scale } \\
\hline Score 1-3 & $18(25 \%)$ & $9(27 \%)$ & $7(29 \%)$ & $34(79 \%)$ & $34(26 \%)$ \\
\hline Score $>3$ & $9(13 \%)$ & - & - & $9(21 \%)$ & $9(7 \%)$ \\
\hline Patients with underlying CKD (\%) & $7(10 \%)$ & - & - & $7(16 \%)$ & $7(5 \%)$ \\
\hline Mortality (\%) & $13(18 \%)$ & $5(15 \%)$ & $2(8 \%)$ & $20(47 \%)$ & $20(15.5 \%)$ \\
\hline Relative risk versus CPAP & - & 1.6 & 1.4 & - & - \\
\hline $95 \%$ confidence interval & - & $(0.76-3.28)$ & $(0.49-4.16)$ & - & - \\
\hline
\end{tabular}

\section{Background}

Acute kidney injury (AKI) has been associated with increased mortality and morbidity in COVID-19 patients. ${ }^{1-3}$ It is hypothesised to be worse in patients with underlying chronic kidney disease (CKD). The aetiology of AKI in these patients is multifactorial, with direct cytopathic effects on kidney tissue, involvement of angiotensin-converting enzyme 2 and immunemediated coagulopathy all playing a role. ${ }^{2}$ Although some studies have shown an association between kidney involvement and poor outcome in COVID-19, information on this subject remains limited. ${ }^{1-3}$ We evaluated the prevalence of AKI in a subgroup of patients with COVID-19 and the associated mortality.

\section{Method}

This was a prospective cohort study of 130 patients with COVID-19 admitted to respiratory high dependency (HDU) or intensive care (ITU) settings. Renal dysfunction, respiratory support requirements and mortality were the clinical parameters of interest.

Author: ${ }^{A}$ Gloucestershire Hospitals NHS Foundation Trust, Gloucester, UK

\section{Results}

$28.4 \%$ of patients were female with median age of 60 years (range $22-88$ years). $33 \%(n=43)$ of patients were diagnosed with AKI on admission, with $16 \%(\mathrm{n}=7)$ of these known to have underlying CKD. One patient had a previous renal transplant. $6 \%$ of patients had hypertension and $3 \%$ of patients were known diabetics. Overall mortality was $15.5 \%(\mathrm{n}=20)$ in patients with AKI on admission, with the majority $(10 \%, n=13)$ seen in the CPAP cohort likely attributable to underlying CKD (Table 1).

\section{Discussion}

Patients with AKI on admission were more likely to be admitted to ITU and to undergo mechanical ventilation compared with patients with underlying CKD, suggesting that AKI on admission represents a higher risk of deterioration and overall mortality.

Our data show higher mortality in patients with low clinical frailty, which may represent the cohort selection bias for HDU/ ITU. This observation indicates poor prognosis regardless of clinical frailty in COVID-19 patients diagnosed with AKI. Monitoring renal function must therefore be emphasised in all patients requiring admission with declining renal function.

Early detection and treatment in patients with comorbidities associated with renal decline such as hypertension and diabetes may help improve prognosis in COVID-19. 


\section{Conflicts of interest}

None declared.

\section{References}

1 Cheng Y, Luo R, Wang K et al. Kidney disease is associated with inhospital death of patients with COVID-19. Kidney Int 2020;97:829-38.
2 Hirsch JS, Ng JH, Ross DW et al. COVID-19 Research Consortium; Northwell Nephrology COVID-19 Research Consortium. Acute kidney injury in patients hospitalized with COVID-19. Kidney Int 2020;98:209-18.

3 Oliveira CB, Lima CAD, Vajgel G et al. High burden of acute kidney injury in COVID-19 pandemic: systematic review and meta-analysis. J Clin Pathol 2020, in press (DOI: 10.1136/ jclinpath-2020-207023). 